Théologiques

Théologiques

\title{
L'initiation : sens et défis sur l'horizon de la mission catéchétique de l'Église
}

\section{Solange Lefebvre}

Volume 9, numéro 1, printemps 2001

Les cosmologies

URI : https://id.erudit.org/iderudit/005688ar

DOI : https://doi.org/10.7202/005688ar

Aller au sommaire du numéro

\section{Éditeur(s)}

Faculté de théologie de l'Université de Montréal

\section{ISSN}

1188-7109 (imprimé)

1492-1413 (numérique)

Découvrir la revue

\section{Citer cet article}

Lefebvre, S. (2001). L'initiation : sens et défis sur l'horizon de la mission catéchétique de l'Église. Théologiques, 9(1), 143-164.

https://doi.org/10.7202/005688ar
Résumé de l'article

Comment réfléchir aujourd'hui sur l'initiation en christianisme ? L'anthropologie renvoie à un sens de l'initiation plus précis que ne le fait le champ de l'initiation sacramentelle, très large. Et ce sens fondamental se rapporte notamment à la distinction entre rites d'initiation et rites de passage. Les premiers se fondent dans le processus d'incorporation proprement rituelle. Cela dit, il reste que l'approche de l'initiation dans nos sociétés modernes se présente plus généralement comme un chemin de sens évoquant de loin et par traces les antiques expériences initiatiques. L'enjeu est de saisir comment ces expériences continuent de travailler les quêtes religieuses et chrétiennes actuelles. 


\title{
L'initiation : sens et défis sur l'horizon de la mission catéchétique de l'Église ${ }^{1}$
}

\author{
Solange LeFEBVRE \\ Faculté de Théologie \\ Université de Montréal
}

Cet article s'inscrit dans le champ de la réflexion sur les défis catéchétiques rencontrés par l'Église québécoise. Dans un premier temps, quelques remarques de départ sont élaborées au sujet de la pertinence et des limites de la question de l'initiation, au plan anthropologique et théologique, pour les communautés chrétiennes. Vient ensuite une clarification de la distinction entre rites d'initiation et rites de passage, importante à faire dans le contexte actuel. Une troisième partie aborde le pari de l'initiation comme chemin de sens dans la modernité. Quelques formulations de propositions ou enjeux permettent de conclure.

\section{Pertinence de l'enjeu initiatique}

Avant d'aborder plus explicitement le thème de l'initiation, il faut faire quelques remarques préalables. Les journées de réflexion de l'A.E.Q. de mars 2001 avaient pour thème précis l'initiation chrétienne dans la culture actuelle. Pourquoi ce choix ? Le comité préparatoire a certes ses raisons, notamment celle-ci : dans le champ touffu de l'éducation de la foi, il fallait cibler un thème précis. J'ajouterais que cette question clé renvoie à un enjeu fondamental, évoqué par Abel Pasquier, cet ethnologue qui réfléchit aussi sur l'initiation chrétienne : «La santé d'un groupe social (et/ou religieux) risque sans cesse d'être compromise par les aléas de la subjectivité des destins individuels. Aussi s'évalue-t-elle par sa capacité à incorporer rituellement de nou-

1. Ce texte développe les éléments exposés brièvement devant l'Assemblée des évêques du Québec et leurs collaborateurs et collaboratrices, durant la session du mois de mars 2001. 
veaux membres ${ }^{2}$ ». Or, cette incorporation rituelle réfère précisément à la dimension initiatique.

Au sein du vaste défi de l'éducation de la foi, la question de l'initiation est donc centrale. J'entrevois à ce sujet un double défi : 1) clarifier les rapports entre initiation et rites de passage ; 2) poursuivre un travail d'intelligence initiatique afin d'être capable de ressaisir les éléments les plus marquants et les plus forts d'une éducation de la foi. Pour répondre à ce double défi, j'élabore quelques éléments de réflexion sur ce vaste sujet. Mais j'aimerais situer la présente réflexion.

Mon intérêt pour la question de l'initiation date de 1991-1992, année où parut le premier livre de la recherche action de notre équipe dirigée par Jacques Grand'Maison, dans le diocèse de Saint-Jérôme : Le drame spirituel des adolescents ${ }^{3}$. Ce livre allait connaître un succès qui ne s'est pas démenti, non seulement dans les milieux pastoraux, ecclésiaux et chrétiens, mais aussi dans de nombreux milieux professionnels et académiques. Ce sont en particulier les thèmes de la transmission, de l'initiation, des rapports de générations et des valeurs qui suscitèrent l'intérêt.

Le thème de l'initiation était donc abordé dans ce rapport de recherche, comme une piste pastorale et culturelle prometteuse qu'il nous fallait revisiter. Nous soulevions l'enjeu socio-culturel bien connu de la jeunesse comme passage, c'est-à-dire une expérience forte et profonde, parfois trouble, de mort et de vie, voire comme un enjeu pascal. À l'instar d'autres auteurs, nous distinguions trois façons de transmettre: l'enseignement d'un contenu, l'apprentissage d'un contenu et d'un savoir-faire, l'initiation qui nous paraissait englober les deux autres, en plus du savoir-être (symboles, histoire, aspirations, valeurs, etc.).

Nous observions que les jeunes, privés de lieux initiatiques d'apprentissage et de resymbolisation, se donnaient à eux-mêmes des ersatz ou des substituts d'initiation. Nous proposions certaines démarches initiatiques originales, qui tenaient compte du sens symbolique

2. A. Pasquier, "Identification de rites initiatiques contemporains ", dans Bernard KAEMPF (dir.), Rites et ritualités, Cerf/Lumen Vitae/Novalis, 2000, p. 176-177.

3. Jacques GRAND' MaIsOn, Le drame spirituel des adolescents. Profils sociaux et religieux. Montréal, Fides, 1992. 
des jeunes, de leur quête de valeurs, de leur effervescence imaginaire ; et qui respectaient à la fois leur dynamique de l'entre-pairs et leur besoin de modèles adultes structurants.

Ces aspects reçurent une réception étonnante dans une grande diversité de milieux. Il a bien fallu affiner la réflexion, répondre à de nombreuses questions théoriques et pratiques. L'un de mes champs de recherche étant celui des rapports de générations, je pus développer diverses pistes en ce sens, tout en travaillant en collaboration avec des collègues d'autres disciplines sur des enjeux de transmission. L'initiation est en effet à situer au sein du vaste chantier de réflexion sur ce dernier thème.

\section{Initiation : approche anthropologique}

Lorsqu'il s'agit de situer la question de l'initiation à la fois au sens anthropologique et théologique, un premier problème se pose. $\mathrm{La}$ définition anthropologique est beaucoup plus limitée que ne l'est la définition théologique. Celle-ci est en effet devenue au fil du temps tellement large qu'on ne peut plus guère parler de définition mais plutôt d'approche. La raison en est simple : l'anthropologie, même si elle s'attache à présent à l'étude des sociétés occidentales, conserve son ancrage principal dans des sociétés non occidentales, où les schèmes d'initiation - s'il en est - demeurent plus clairs. La modernité occidentale a un impact considérable sur l'expérience religieuse, dans le sens d'une fragmentation et d'une pluralisation qui font échapper la majeure partie des individus à une expérience initiatique au sens strict, c'est-à-dire forte, discontinue et irréversible.

Pour se convaincre de cette différence, on n'a qu'à lire les écrits sur l'initiation chrétienne des trente dernières années, et les comparer aux écrits anthropologiques. Les premiers utilisent le terme "initiation" dans plusieurs sens : initiation comme formation générale, initiation comme démarche globale de foi, initiation comme rite, etc. Pour reprendre la distinction généralement reconnue, on rencontre l'initiation chrétienne comme processus global, et l'initiation par et à travers les rituels ou les sacrements ${ }^{4}$. Cette dernière signification, rattachée

4. Au sujet de ces débats, voir Marie-Josée POIRÉ, « La notion d'initiation chrétienne et le Rituel de l'initiation chrétienne des adultes »: Liturgie, Foi et Culture, vol. 33, hiver 1999, p.21-32. 
aux premiers siècles du christianisme, est plus proche du sens anthropologique.

Que peut-on en dire au sens anthropologique ? Il y aurait trois types d'initiation : 1) les initiations tribales des garçons, plus rarement des filles, au statut d'adulte de plein droit (ou rites de puberté) ; 2) les initiations religieuses des intermédiaires choisis entre le monde humain et les puissances invisibles (prêtres, devins, possédés, chamanes, etc. ; initiations mystiques) ; 3 ) les initiations volontaires aux sociétés ou confréries secrètes. Pour appréhender la radicalité de l'initiation au sens anthropologique, décrivons un peu les rites de puberté, à la fois sociaux et religieux. Les cérémonies d'initiation comportent généralement les éléments suivants :

1) préparation d'un terrain sacré où s'isoleront les hommes pendant la durée de la fête ;

2) séparation des novices de leur mère ;

3) isolement, parfois prolongé, dans un lieu retiré où ils seront instruits des traditions religieuses de la tribu ;

4) imposition au novice d'épreuves initiatiques plus ou moins cruelles (circoncision, tatouage, scarification, arrachage des cheveux, épreuve du feu, extraction d'une dent);

5) retour du nouvel initié dans son groupe et célébrations de son nouveau statut d'adulte mis au service du groupe.

Il s'y trouve trois aspects fondamentaux:

1) la séparation du monde féminin et de celui de l'enfance (thème de la mort)

2) marge : introduction au sacré et épreuves

3) régénération collective de la société et festivités

Peut-on reconnaître dans les trois formes d'initiation signalées plus haut des traits communs ? Il est courant de référer à Arnold Van Gennep (1909) pour mettre en évidence que les rites de passage, dont l'initiation serait la meilleure illustration, comportent trois phases : séparation, marginalisation (mise à l'épreuve), agrégation ${ }^{5}$. Initier serait essentiellement faire passer un individu ou un groupe d'un état ou d'un statut à un autre, à travers ces trois étapes. Or, dans les faits, l'initiation ne se déroule pas nécessairement en trois étapes, et surtout,

5. Arnold Van Gennep, Les rites de passage, Paris, 1909, Émile Nourry. 
sa particularité n'est pas de faire passer d'un état à un autre, ni de marquer un passage de la puberté à l'âge adulte ou la différence des sexes, mais celle-ci : à la différence des rites de passage qui marquent la naissance, la puberté, la mort ou d'autres étapes, "l'initiation consiste à engendrer une identité sociale au moyen d'un rituel et à ériger ce rituel en fondement axiomatique de l'identité sociale qu'il produit ${ }^{6}$ ".

Au cœur de cette nouvelle identité se trouve la dimension d'une « vie nouvelle ", qui rend l'initié apte à son tour à être agent d'initiation. L'ethnologie conçoit donc l'initiation comme suit : « ni un simple rite de transition ni un processus d'apprentissage continu mais un rite de formation discontinue et irréversible. " L'initiation brise le rythme continu de l'existence. De plus, ce rite de formation promeut la personne en représentant d'une catégorie sociale particulière. La discontinuité est le plus souvent symbolisée à travers les rites de la mort et de la renaissance des néophytes (avalés par des ancêtres, tués par les esprits, soumis à un risque de mort, soumis à un rituel d'anéantissement, etc).

De plus, la transformation initiatique s'accompagne souvent de la barrière du secret et d'un jeu subtil qui fait en sorte qu'on établit une distance entre l'initié et le non-initié, selon le schéma dehors dedans. L'initiation crée un groupe qui se forme par exclusion des non-initiés. Par ailleurs, dans les initiations traditionnelles masculines, on trouve toujours une légitimation de la subordination politique et économique des femmes aux hommes ; on érige en principe cosmique la domination masculine. Et les rites d'initiation, dans ce cas, s'approprient symboliquement certains attributs féminins.

L'initiation est en quelque sorte le rite des rites, - concentré des rites de passage au moyen desquels elle opère - , car elle définit l'identité des agents, qui assurent sa reproduction. Dans ce but, elle utilise des procédés d'épreuves et de marquages physiques : circoncision, percement du nez, etc. Ces opérations douloureuses contraignent la mémorisation de l'initiation mais surtout « naturalisent une transfor-

6. Voir A. ZEMPLÉNI, "Initiation ", dans Pierre Bonte et Michel Izard (dir.), Dictionnaire de l'ethnologie et de l'anthropologie, Paris, PUF, 1991, p. 375-377. 
mation avant tout culturelle ". Faisons aussi ressortir les traits suivants : la dimension de mort symbolique ou mystique - « Mourir c'est être initié ", disait Platon ${ }^{7}$-, le secret, la transmission d'un initié à un autre, le clivage dedans - dehors.

Mircea Eliade écrit ceci :

L'intérêt de l'initiation pour l'intelligence de la mentalité archaïque réside surtout en ceci : elle nous montre que le vrai homme, — l'homme spirituel —, n'est pas donné, n'est pas le résultat d'un processus naturel. Il est «fait » par les vieux maîtres, selon les modèles révélés par les Êtres divins et conservés dans les mythes. Ces vieux maîtres constituent les élites spirituelles des sociétés archaïques. Eux savent, eux connaissent le monde de l'esprit, le monde véritablement humain. Leur fonction est de révéler aux nouvelles générations le sens profond de l'existence et de les aider à assumer la responsabilité d'être un " homme véritable ", et, par conséquent, de participer à la culture. Mais puisque, pour les sociétés archaïques, la " culture " est la somme des valeurs reçues des Êtres surnaturels, la fonction de l'initiation peut se ramener à ceci : elle révèle, à chaque nouvelle génération, un monde ouvert vers le trans-humain, un monde, dirions-nous, transcendantal ${ }^{8}$.

On voit donc que l'initiation traditionnelle revêt un sens très précis de transformation de l'individu selon les règles du groupe auquel il appartient. Qu'elle provoque une logique d'exclusion des non-initiés, qu'elle se fait à travers un rituel, qu'elle transforme fortement l'identité même de l'initié à travers ce rituel. Elle est en outre inscrite à l'intérieur des structures traditionnelles de parenté et de rapports entre les sexes. Elle renvoie aux rites de passage mais s'en distingue aussi. Nous verrons plus loin ce qu'il en est de l'initiation, reprise à nouveaux frais dans les réflexions plus contemporaines. Mais attardons-nous d'abord sur l'importante distinction entre initiation et rite de passage.

7. Les Grecs faisaient d'ailleurs le jeu de mots suivant : teleîsthai - être initié ; teleutân - mort. Voir Mircea ELIADE, Initiation, rites, sociétés secrètes, Paris, Folio/Essais 196, Gallimard, 1959.

8. Ibid., p. 277. 


\subsection{Initiation et rite de passage : comment différencier ces notions?}

Les insatisfactions des chrétiens et chrétiennes engagés se rattachent souvent aux investissements pastoraux massifs dans les rites d'initiation chrétienne - baptême, première communion, confirmation : "Puisque les enfants et les parents ne reviennent pas à l'Église après, suggèrent-on alors, il faudrait conditionner l'accès aux rites d'initiation à une véritable intention de s'engager dans un itinéraire de foi adulte. »Cet argument risque d'être renforcé par la nouvelle demande catéchétique, après un certain retrait de l'école en matière d'éducation chrétienne. Devant le défi accru d'éducation de la foi, on pensera peut-être à recentrer les efforts de tous et toutes.

La demande d'initiation chrétienne perdure et on en a maintes fois analysé les raisons, surtout au niveau sociologique : il peut s'agir d'une inscription ponctuelle dans une tradition familiale et religieuse, d'un besoin de références stables et d'un ancrage dans le temps liturgique au sein d'un monde précaire à bien des titres (relations, statuts sociaux, problèmes collectifs, etc.), d'une foi individuelle qui choisit de se rapporter, le temps du rituel seulement, à une vaste communauté de référence, d'un simple phénomène social d'entraînement, etc.

Plus récemment, dans la foulée des travaux de Danièle HervieuLéger, on paraît s'entendre autour du fait que les cadres collectifs de la mémoire collective se sont effondrés, et que l'une des conséquences est une crise de la transmission. Le statut de la tradition s'est transformé, les raisons de s'y inscrire diffèrent d'une famille à l'autre. Qu'on se le dise, il serait devenu quasiment impossible de transmettre la foi chrétienne dans toutes ses dimensions?

On ne peut nier que ces analyses sociologiques ne satisfont pas toujours les chrétiens et les chrétiennes qui accueillent et accompagnent les gens sur le terrain. Ils font souvent face à une déculturation religieuse et chrétienne décourageante, à des attitudes ambivalentes. Il me semble qu'il faut évoquer la distinction entre rites de passage et

9. Danièle HERVIEU-LÉGER, La religion pour mémoire, Paris, Cerf, 1993 ; cf. Solange LEFEBVRE, "La crise de la transmission et son dépassement » : Église de Saint-Jérôme, vol. 34 no 1, janvier-février 1996, p. 9-11; et aussi "Quotidien et foi chrétienne. Aspects anthropo-théologique » : Liturgie, Foi et Culture, vol. 32, Printemps 1998, p. 3-9. 
initiation, afin de bien nommer ces frustrations, de bien situer le contexte et les limites de nos efforts pastoraux.

Si l'initiation au sens anthropologique réfère à un changement radical de statut à l'intérieur d'un rite bien conduit, et au sens chrétien à une démarche graduelle d'incorporation à la communauté, qu'en est-il des rites de passage?

Comme nous l'avons écrit plus haut, les rites de passage concernent des réalités beaucoup plus larges que l'initiation proprement dite. Van Gennep avait fait l'étude systématique des rites de la porte et du seuil, de l'hospitalité, de l'adoption, de la grossesse et de l'accouchement, de la naissance, de l'enfance, de la puberté, de l'initiation, de l'ordination, du couronnement, des fiançailles et du mariage, des funérailles, des saisons, etc. Et nulle approche de ces questions ne peut faire l'économie d'une référence à cet auteur.

Rappelons ce qui caractérise les rites de passage. D'abord s'y succèdent dans le temps trois stades : séparation, marge, agrégation (dits aussi préliminaires, liminaires et postliminaires - du latin limen, seuil). Ensuite ils se caractérisent par leur matérialité, c'est-à-dire par le fait qu'ils se vivent de manière matérielle et physique très concrète, et rarement de façon uniquement symbolique. On passera donc à travers une porte, sur un chemin, d'une pièce à l'autre, d'un vêtement à l'autre, etc. Et il est courant de rapporter tous ces passages au passage premier universel, c'est-à-dire la naissance, " premier passage matériel que tout être humain doit subir pour devenir tel ${ }^{10}$ ".

En troisième lieu, ils s'ancrent dans la réalité biologique de la vie. Van Gennep relie étroitement les rites de passage au déroulement de la vie individuelle et collective, voire au "fait même de vivre ", ce qui renvoie à ces propos de Lévi-Strauss : "l'ordre social et religieux non seulement a quelque chose à voir avec les faits de l'ordre biologique, mais aussi cherche à les traduire, les expliquer, en tirer parti, les exploiter ${ }^{11} »$.

10. Voir Nicole BeLmont, "La notion du rite de passage ", dans P. CENTLIVRES et J. HAINARD (dir.), Les rites de passage aujourd'hui, Lausanne, L'Age d'Homme, 1986, p.16.

11. Ibid. Cite C. LÉVI-STRAUSS, "Art, passage, rites ", in : Encyclopaedia britannica, vol. 17, p. 354. 
Dans les textes concernant l'initiation chrétienne, on parle parfois indifféremment de rites d'initiation et de rites de passage. Nous avons vu en quoi les rites d'initiation sont particuliers, au sein des nombreux rites de passage. Mais ils en conservent aussi certains traits. Quelles sont les conséquences pour l'initiation chrétienne ? Il y a un lien intime entre rites de passage - au sens culturel et social - et initiation chrétienne proprement dite. Cela signifie que la signification religieuse et chrétienne peut même être absente chez certains sujets, dont la demande demeure culturellement et socialement légitime. Notons cependant que cet écart parfois radical entre rites de passage culturels et initiation religieuse caractérise les sociétés sécularisées. En tant que grande tradition religieuse historique, le catholicisme a dû forcément répondre à des demandes proprement anthropologiques, culturelles et sociales. Il a forcément des enracinements socio-culturels qui précèdent et accompagnent l'expérience et la décision de la foi. Il a quelque chose à voir avec la naissance, avec les jalons de l'enfance et de la vie familiale. Il ponctue le rythme biologique de la vie, il ne concerne pas exclusivement le credo dans la mort et la résurrection de Jésus-Christ, et l'adhésion engagée dans une communauté de foi qui professe celleci. Aussi faudra-t-il vivre la réalité de la demande et de l'expérience pastorale des rites d'initiation chrétienne durant l'enfance au coeur même de la tension entre l'initiation comme engagement de foi, et le rite de passage comme marquage matériel et rituel d'une étape de vie. Notre monde pastoral a donc besoin d'une intelligence à la fois théologique et anthropologique ou culturelle. Les deux dimensions - rite d'initiation et rite de passage - sont porteuses d'un sens. Cela dit, il faut certes clarifier la spécificité chrétienne des rites.

\section{Un pari sur l'initiation comme chemin de sens dans la modernité}

Mircea Éliade, ce chrétien orthodoxe d'origine roumaine qui s'est intéressé aux expériences universelles et plurielles de la religion, évoque toujours la modernité comme un lieu de dissolution du sens religieux de la vie humaine. Eu égard à l'initiation, il constate que le monde moderne ne connaît plus d'initiation de type traditionnel. Mais il offre une série de catégories très utiles pour saisir les traces ou les équivalents de scénarios initiatiques en modernité. Éliade, dans la foulée de Carl Jung, fait partie des auteurs qui estiment que le psychisme humain aspire d'une quelconque manière à l'initiation reli- 
gieuse. Ces approches sont certes mises en question, on le verra dans la partie suivante. Mais il me paraît qu'elles renvoient à des expériences réelles d'attrait pour l'initiation dans notre culture.

\subsection{Horizon bistorique}

Si l'on se reporte à la définition de l'initiation au sens anthropologique évoquée plus haut, déjà en Grèce antique on assiste à une certaine atténuation des rites d'initiation, par exemple à travers les rites de puberté qui permettent d'initier à l'éducation civique. De manière non dramatique, on initie des garçons à la vie religieuse de la cité. Le type de vocabulaire dont use Éliade me semble pertinent pour nous : on ne trouverait désormais que des "dérivés " ou des "survivances " d'un scénario initiatique, des "réactivations " de thèmes et de traits initiatiques. Et voici son explication d'inspiration jungienne :

C'est comme si les scénarios de l'initiation étaient indissolublement liés à la structure même de la vie spirituelle. Comme si l'initiation était un processus indispensable à tout essai de régénération totale, à tout effort de transcender la condition naturelle de l'homme afin d'accéder à un mode d'être sanctifié ${ }^{12}$.

Retenons de l'époque grecque par exemple les récits d'histoire de héros ou de dieux soumis à de longues épreuves, jusqu'à la renaissance humaine et spirituelle, et de même l'assimilation de la formation philosophique à l'initiation, à travers la notion de « maïeutique » (cf. maïa, " accoucheuse »), selon laquelle Socrate accouchait ses disciples comme des hommes nouveaux, renvoyant de la sorte au travail des maîtres archaïques qui accouchaient leurs disciples à une vie spirituelle. De là est aussi issu le thème de "paternité spirituelle », repris par le brahmanisme, le bouddhisme et le christianisme. Saint Paul par exemple évoque ses " fils spirituels " qu'il a engendrés à la foi.

Qu'en est-il des débuts du christianisme ? Y trouve-t-on des éléments initiatiques ? Oui, dans les rituels du baptême et de l'eucharistie. Les dimensions suivantes y sont présentes : introduction dans une nouvelle communauté (dehors, païens - dedans, élus), nouvelle identité spirituelle et sanctification de l'initié qui modifient radicalement son existence, promesse de vie éternelle, bains rituels, banquets

12. Ibid., p. 246. 
rituels. La Résurrection de Jésus-Christ est elle-même de texture initiatique ; elle introduit une rupture/discontinuité dans l'histoire, en lui il y a transmutation spirituelle de l'être humain et rénovation du monde. Il s'agit aussi d'un mystère (élément initiatique) mais au début, sans le secret. L'initiation chrétienne est accessible à tous et toutes. Dans les écrits de Paul on note ce déplacement : le mystère ne réfère pas à un scénario initiatique secret des religions antiques mais au secret de Dieu qui se trouve dans son intention de sauver les êtres humains par Jésus-Christ. De la sorte, on se démarque des initiations païennes.

Peu à peu, ce christianisme primitif reprendra davantage d'éléments initiatiques : symboles archaïques (arbre de vie et croix chez les Pères de l'Église), emprunt aux thèmes des Mystères grecs, reprise de thèmes de la philosophie grecque. On insiste davantage sur la mort symbolique et mystique procurée par le baptême, liée à celle du Christ et, plus tard, la dimension secrète est remise de l'avant. Mais c'est par l'intermédiaire des sacrements chrétiens qu'on initie aux mystères. Souvent il est reconnu par ailleurs que le christianisme a attaqué les mythes et, en se proclamant religion de salut accessible à tous, il s'est progressivement dégagé de l'atmosphère et des rites du mystère initiatique.

Par la suite, jusqu'à aujourd'hui, on trouve des survivances et des mutations successives de scénarios initiatiques dans l'Europe chrétienne, du Moyen Âge à l'âge moderne. J'en retiens ce qui peut éclairer notre actualité. Certaines coutumes folkoriques en conservent des traces - les carnavals ou les mascarades (pensons à notre Halloween), même si elles ont oublié leur genèse initiatique. Certains rites masculins de puberté se trouvent encore dans l'Europe rurale du XIX ${ }^{\mathrm{e}}$ siècle, sans la dimension de mort et de renaissance spirituelles. Ajoutons à cet égard certains rites dans l'armée. Les corporations de métiers reprennent d'anciens thèmes initiatiques : secrets du métier, relation étroite maître-apprenti, étapes de probation, serment de silence sur les secrets du groupe (maçons, forgerons ...).

Quant aux cercles fermés d'initiés, on en trouve un petit nombre. Celui des alchimistes, par exemple, qui répercutent la symbolique épreuve-mort-résurrection dans la Matière elle-même. Dans les documents rapportant les procès des Templiers et des sorcières au Moyen Âge, on trouve des récits évoquant leurs scénarios très secrets de nature initiatique. 
Un élément mérite notre attention particulière. Éliade note l'émergence au XII ${ }^{\mathrm{e}}$ siècle, d'une littérature à succès truffée de scénarios initiatiques, autour du thème du Roi Arthur et de la quête du Graal, d'origine celtique. Ces récits présentent les traits suivants : une quête longue et pleine d'embûches, la recherche d'objets merveilleux, la pénétration du héros dans un autre monde, la régénération du Royaume d'Arthur par le triomphe du héros. Permettez moi ici de citer de nouveau Éliade car son observation de la diffusion massive des «motifs littéraires" au Moyen Âge vaut, me semble-t-il, pour aujourd'hui :

Le fait qu'on écoutait avec délices des histoires romanesques où les clichés initiatiques revenaient à satiété, prouve, il nous semble, que de telles aventures répondaient à un besoin profond de l'homme médiéval. Les scénarios initiatiques n'alimentaient plus que l'imagination, mais la vie imaginaire, comme la vie onirique, est aussi importante pour la totalité psychique de l'être humain que la vie diurne (c'est le problème du psychologue) ... il faut toucher (ce problème) pour comprendre ce que sont devenus la majorité des scénarios initiatiques, lorsqu'ils eurent perdu leur réalité rituelle : ils sont devenus ce qu'on les trouve, par exemple, dans les romans arthuriens : des "motifs littéraires ». C'est dire qu'ils délivrent maintenant leur message spirituel sur un autre plan de l'expérience humaine, en s'adressant directement à l'imagination ${ }^{13}$.

Et il en va de même des contes de fées. Les scénarios initiatiques dont s'abreuvent sans répit les humains - même camouflés - semblent l'expression d'un psychodrame répondant à une nécessité profonde. À partir du Moyen Âge donc, l'art et la littérature deviennent le moyen par excellence pour transmettre et communiquer une théologie, une métaphysique, une sotériologie. Et cela vaut encore aujourd'hui, alors qu'on trouve une profusion de romans initiatiques à succès (pensons à l'Alchimiste), de films à succès (Star Wars, Ninja Turtles, Le cinquième élément et l'idée de régénération par la Femme et l'Amour, et autres films présentant clairement des traces et des éléments de scénarios initiatiques) ainsi que les jeux vidéos et informatiques à mystères. Des oeuvres d'art aussi reprennent les grands traits des initiations. Retenons donc qu'une dominante de l'ère moderne, en ce qui a trait à l'initiation, est le fait qu'on met en scène pour l'imagination des scénarios initiatiques.

13. Mircea ElIADE, Ibid., p. 266-267. 
En outre, nos sociétés sécularisées conservent des éléments initiatiques dans les cérémonies religieuses, certaines cérémonies civiles, dans des compétitions sportives, dans des spectacles, dans des publicités, souvent sans contenu religieux. Autre lieu de survivance initiatique que signale Eliade : les mouvements religieux qui se rapportent à des schémas initiatiques, et qui jouent souvent une fonction positive même s'ils n'offrent pas une grande complexité et richesse spirituelle.

De manière analogue, la succession des étapes et des cycles de vie conserve une logique initiatique, car on devient humain à travers une série d'épreuves, de morts et de renaissances. Cela est reconnu sans qu'on y voit une signification religieuse, sans la transformation ontologique et identitaire radicale, sans la discontinuité radicale que présentaient les scénarios initiatiques au sens strict. Mais ceux-ci imprègnent toujours le psychisme de l'être humain. Le sujet contemporain subjectif et esthétique cherche l'évasion et la distraction dans des réalisations à thématiques initiatiques (livres, films, jeux). Il fait face, comme les anciens, aux défis de morts et de vie, au désir d'immortalité ; il peut chercher à divers moments à obtenir un renouvellement radical de sa vie : "C'est à une telle renovatio qu'aboutit toute conversion religieuse authentique ${ }^{14}{ }$.

\subsection{Deux grandes positions}

Éliade appartient donc aux auteurs qui soutiennent que l'être humain a un besoin profond d'initiation. On peut situer dans cette foulée Victor Turner, Philippe Emmanuel Rausis, Jacques Grand'Maison et coll., Abel Pasquier et plusieurs autres. ${ }^{15}$

J'estime que cette analyse est utile pour nos milieux. Mais plusieurs autres auteurs refusent de nommer "rite d'initiation » ou "rite de passage ", des semblants ou des traces partielles qu'on en trouve dans la modernité. Leur mérite est de faire valoir les différences fondamentales que présentent les expériences actuelles. Les principales critiques

14. EliAde, Ibid., p.282.

15. Voir Pierre-Emmanuel Rausis, L'initiation [Bref,45], Paris, Cerf/Fides, 1993 ; J. Grand-Maison, A. Durocher et S. Lefebvre, "Partie 4 : L'initiation : une pratique fondamentale à renouveler ", dans J. GRAND' MAISON, L. BARONI et J.-M. GAUTHIER (dir.), Le défi des générations, Montréal, Fides, 1995 ; PASQUIER, loc.cit. 
de l'utilisation de la catégorie d'initiation et aussi de rite de passage dans les sociétés modernes s'articulent de la manière suivante :

Le symbole identitaire du rite de passage a souvent créé la tentation de l'appliquer aux sociétés modernes, notamment à l'adolescence ou même à la retraite. Ces transpositions sont abusives car les transitions dans les sociétés modernes sont graduelles, variables, différenciées ; elles sont en outre individualisées et marquent la continuité de l'existence autant que ses ruptures, à la différence du rituel de passage qui renforce l'identification collective et met l'accent sur la coupure entre les âges ${ }^{16}$.

Effectivement, la période de la jeunesse s'est allongée, les étapes de transition se sont brouillées, multipliées et désynchronisées, le passage n'est plus structuré par des rites de passage mais des processus uniformes et administratifs. On trouve plutôt de nombreux rites qui président à des évolutions ponctuelles, réversibles et symboliques, alors que les rites de passage organisaient un changement complet, irréversible et solennel. Cette mutation repose sur une modification des rapports de générations, un déclin des communautés d'interconnaissance et donc du contrôle d'une génération sur une autre ; l'allongement des transitions et l'apparition de rites ponctuels qu'on intitulera " rites de première fois ": premier rapport sexuel, début de la vie en couple, fin des études, accès à l'indépendance résidentielle, premier emploi stable, premier enfant et mariage ${ }^{17}$.

Le récent document de l'Assemblée des évêques du Québec, intitulé "Proposer aujourd'hui la foi aux jeunes ", prend acte de ces mutations, tout en se situant dans une logique initiatique. La dynamique du document est intéressante. Tout un vocabulaire s'y déploie, du type : parcours, chemin, itinéraire. On y propose ceci : "C'est l'ensemble de ces chemins qu'il convient de proposer aux jeunes. Comme des chemins d'initiation, c'est-à-dire des chemins d'introduction, de premier contact, de premier apprentissage. Comme autant de voies d'accès possibles à l'expérience chrétienne ${ }^{18}$.

16. Claudine ATTIAS-DONFUR, Générations et âges de la vie [Que sais-je ?], Paris, PUF, 1991, p. 44.

17. Voir Michel Bozon, « Des rites de passages aux "première fois". Socioethnologie des rites de la jeunesse en France ", manuscrit, 13 p.

18. AEQ, Proposer aujourd'hui la foi aux jeunes. Une force pour vivre, Montréal, Fides, 2000, p. 25. 
Dans les milieux théologiques, il m'apparaît pertinent d'accorder beaucoup d'attention aux réalités fondamentales d'initiation et de rite de passage, tout en gardant ces notions ouvertes, flexibles. Elles nous semblent pédagogiquement précieuses pour répondre au besoin chez l'être humain de marquer des étapes, de vivre des moments où il peut ressaisir et resymboliser les dimensions à la fois humaine, morale et spirituelle de sa vie, avec une ouverture vers la collectivité.

Voici un exemple de rite de passage moderne: dans certaines universités où l'on avait délaissé les cérémonies de graduation, on s'y remet, avec costumes, solennité, représentations hiérarchiques et institutionnelles, discours et le reste. De la sorte, on introduit le diplômé dans l'univers professionnel, à un nouveau statut, avec ses pairs et devant ses éducateurs et hauts responsables. C'est un moment fort pour tous. Et ce retour des cérémonies de graduation nous renvoie, pour nos milieux ecclésiaux, au besoin de marquages rituels solennels des temps forts. La solennité constitue un marquage matériel et temporel. On ne saurait trop insister sur la qualité musicale, symbolique et liturgique des cérémonies, comme des éléments fondamentaux de tout rite d'initiation chrétienne.

Chez les jeunes, les phénomènes de " gang » ou de " bandes " présentent plusieurs caractères initiatiques, mais à la marge de la société. Des groupes religieux marginaux offriront aussi des scénarios initiatiques. Mais je crois qu'on peut donner crédit à la thèse d'Éliade, selon laquelle les aspirations initiatiques passeraient pour une large part par l'imagination : le roman, le cinéma, les jeux, l'art, etc. Et trait particulier, les films et les jeux destinés aux jeunes surtout mettent souvent en scène un initiateur âgé, qui intervient même depuis l'au-delà pour guider, structurer et protéger le jeune ${ }^{19}$.

À cet égard la filiation entre vieux et jeunes s'avère un élément initiatique que nous revisitons présentement. Après la rupture culturelle entre les générations des années 1960, depuis les années 1990 les jeunes renouent avec les plus âgés. Cela dit, il est important de bien clarifier la nature de cette recomposition sociale des rapports de génération :

19. Ces deux films, parmi d'autres, traitent de l'initiation de plus jeunes par des plus âgés: L'homme sans visage (Mel Gibson), Le facteur (Philippe Noiret). 
1) elle n'est plus unilatérale mais co-éducative, c'est-à-dire qu'elle ne se rapporte plus à un système où les générations plus âgées sont les porteurs uniques d'un savoir à reproduire ; elle se forme autour des savoirs spécifiques à la jeunesse et aux plus expérimentés;

2) elle est élective et affinitaire ; tous les seniors ne sont pas de bons initiateurs et les juniors présentent plusieurs profils d'apprentissage. J'ai forgé à l'intérieur d'une recherche sur les relations de compagnonnage en entreprise les catégories suivantes: jeune hétéronome/adulte ou institution prescripteurs, jeune expérimentateur/ressources ou références ponctuelles, jeune équipier/collectif, protégé/mentor. Je ne vais pas élaborer ces catégories ici mais elles indiquent divers profils éducatifs. Si bien que tous et toutes ne sont pas destinés à nouer des relations fortes sous le mode maître-disciple.

3) la transmission intergénérationnelle se réalise sous des modes divers : récits, exemples, présence, proverbes, conversations, cadeaux, etc. Dans notre enquête sur les aînés, nous avons noté à quel point les plus jeunes s'identifiaient aux aînés, dont les récits de vie renvoyaient à une certaine précarité. De la sorte, ils leur transmettaient un message d'espoir : "Je suis passé à travers, tu peux aussi passer à travers. »

Un autre élément initiatique est le besoin de s'inscrire dans une tradition, même si on ne l'habite plus intensément ou naturellement, d'où le recours aux rites d'initiation chrétienne, les commémorations diverses. Un autre encore réside dans les objets et les symboles. Aux États-Unis, on parle de religion transitionnelle, qui renvoie à l'autonomie personnelle dans la foi :

Il s'agit d'un modèle de religiosité marqué davantage par la quête, la recherche et l'appropriation personnelle, que par le fait d'être et d'appartenir. De plus, la pratique de ce genre de religion semble se faire par la réappropriation de modes d'expériences qui furent traditionnellement réprimés dans notre culture : le corps, l'expérience, les rites de passage, le visuel, les objets ${ }^{20}$.

20. S. LeFEBVRE, "Quotidien et foi chrétienne. Aspects anthropothéologiques ", dans Liturgie, Foi et Culture, Volume 32, Printemps 1998, p. 3-9. 
Parmi les exemples de ces modes d'expérience, retenons celui des rites comme lieux de marquage (mémorisation). Plusieurs églises et groupes religieux américains offrent des parcours initiatiques et des activités sous le symbole du voyage (The Journey). On construit ces journées autour de rites de passage pour les adolescents, en s'inspirant notamment des idées de Joseph Campbell ${ }^{21}$. Celui-ci a été l'inspirateur des films américains mentionnés plus haut, par exemple Star Wars : le héros passe par des épreuves, une formation morale et spirituelle, une découverte de soi et de l'amour. Selon Campbell, « le héros est celui qui sacrifie sa vie à quelque chose de plus grand que lui. » Il y aurait trois phases initiatiques:

1) le héros se sépare de son environnement habituel ; première phase du voyage, du profane au sacré ;

2) aventure initiatique et ses difficultés, épreuves, aide mystérieuse et accès à un source intense de renouvellement se présentant sous le visage d'une divinité ;

3) retour à la vie normale du héros, imbu d'une sagesse nouvelle, partage avec ses semblables les fruits de son aventure spirituelle.

Rappelons que divers mouvements concernés par les jeunes utilisent des schémas initiatiques (scoutisme, Relève, R-Cube ...).

Énumérons brièvement divers lieux séculiers d'inspiration initiatique qui se dessinent actuellement : le néo-aventurier et son risque calculé, qui lui vaut de ressentir la légitimité d'exister, ce que David Lebreton renvoie à l'épreuve médiévale de l'Ordalie ; les rituels sauvages vivaces chez les jeunes (tam-tam du Mont-Royal, perçage et tatouage du corps, voyages initiatiques des jeunes, graffitis, bandes de jeunes, etc. $)^{22}$.

J'ajouterais en terminant que deux figures croyantes actuelles dominantes comportent des éléments initiatiques : le pèlerin et le converti ${ }^{23}$. Le pèlerin renvoie à la marche, au chemin, à la quête, à l'itinéraire, etc. Le converti au sens strict d'une metanoia qui fait culbuter radicalement d'un univers à l'autre offre dans son itinéraire un

21. Joseph CAMPBell, Les héros sont éternels, Paris, Seghers, 1987.

22. Voir David Lebreton, Passions du risque, Paris, Métailié, 1996; et « Rituels sauvages »: Religiologiques 16, automne 1997.

23. Voir Danièle HeRVIEU-LéGER, Le pèlerin et le converti, Paris, Flammarion, 1999. 
schéma initiatique (toujours au sens large). Il témoigne souvent de l'expérience d'une transformation profonde et radicale ; encore fautil savoir l'accompagner pastoralement, pour que son itinéraire se stabilise et s'inscrive bien dans une communauté ${ }^{24}$.

Voilà qui termine ce tour de piste rapide de vastes questions. Je suivrai avec grand intérêt la suite des réflexions de l'Église du Québec sur les nouveaux défis qui se posent à nos communautés chrétiennes. Je risque quelques propositions modestes qui constituent en même temps une sorte de conclusion à ce texte.

\section{Propositions et enjeux}

\subsection{Comment sortir du confinement à l'enfance de l'essentiel de l'édu- cation de la foi ? Accueillir les demandes de ré-initiation ou offrir des démarches en ce sens.}

Plusieurs jeunes et adultes connaissent de riches itinéraires chrétiens. Mais leur nombre est très limité, si l'on compare à celui des enfants engagés dans les rites d'initiation et la catéchèse qui les entoure. Déjà en 1970, dans les débats catéchétiques, on évoquait la possibilité d'un étalement dans le temps, par exemple du sacrement de baptême, "comme un cheminement jalonné par des rites qui aboutiraient à l'accès total à l'expérience baptismale marquant la possibilité de participer à l'Eucharistie ${ }^{25} »$.

Le grand dilemme de la concentration des rites d'initiation durant l'enfance cherche en effet diverses issues : étalement de la démarche dans le temps, report à un âge ultérieur, diversification des démarches

24. Pour aider à comprendre les étapes que traverse la personne convertie, les écueils et les défis d'accompagnement pastoral qui se posent, je vous renvoie à l'excellent ouvrage de Westley Peach, un chrétien évangélique d'origine américaine qui a fait sa thèse de doctorat sur les jeunes adultes convertis, dans le cadre de la recherche-action de Saint-Jérôme. Voir Westley PEACH, Itinéraires de conversion, Montréal, Fides, 2000.

25. Vivement repoussée à l'époque, cette proposition se trouve dans le document suivant : "Le baptême des enfants. Matériaux pour la mise en oenvre de la pastorale ", Office national de liturgie, 1970, p.43; réfère à Guy LAPOINTE, "Quand faut-il baptiser les enfants ? Une voie de recherche " : Liturgie et Vie chrétienne, no 69 (1969), p. 251. 
selon les aspirations, etc. Ces dernières approches concernent des individus particuliers qui présentent une quête de sens et de foi chrétienne plus intense.

Dans le contexte actuel, pourquoi ne pas offrir aussi plus largement une démarche de relance de la démarche initiatique aux parents ou autres accompagnateurs des enfants ? Et ce dans le sens d'un « renouvellement » des promesses, de reprise plus approfondie de la démarche initiale? Si des chrétiens et chrétiennes souhaitent vivre une initiation avec leur enfant au sens initiatique plus strict, on peut de fait leur offrir l'étalement dans le temps ou le report à plus tard, précédé de diverses étapes qualitatives. Mais étant donné le lien intime formé entre rites d'initiation et rites de passage, la majorité des adultes tiendront toujours au parcours classique durant l'enfance. Pourquoi alors ne pas orienter la démarche des adultes vers leur ré-initiation, avec des rites appropriés de renouvellement ? Et de là nous pourrons leur rappeler et leur signifier que l'initié devient initiateur.

\subsection{Tenir à l'enjeu rituel et liturgique comme étant central}

Rappelons ce qui était écrit plus haut, à propos du sens anthropologique de l'initiation : ce qui le différencie le plus des rites de passage est le trait suivant : sa particularité n'est pas de faire passer d'un état à un autre, ni de marquer un passage de la puberté à l'âge adulte ou la différence des sexes, mais celle-ci : à la différence des rites de passage qui marquent la naissance, la puberté ou la mort, "l'initiation consiste à engendrer une identité sociale au moyen d'un rituel et à ériger ce rituel en fondement axiomatique de l'identité sociale qu'il produit. " Même au coeur d'une approche élargie de l'initiation chrétienne, nous ne saurions perdre du vue que les dimensions liturgique et rituelle de l'initiation demeurent centrales.

\subsection{Accepter de vivre au coeur de la tension entre initiation comme en-} gagement de foi et rites de passage comme marquages socioculturels d'une étape de vie. Mais au sein de la demande généralisée des rites de passage, il faut bien sûr demeurer attentifs aux demandes d'initiation proprement dites, chercher à les susciter. 


\subsection{Valoriser et protéger les initiatives particulières \\ et les expériences laboratoires.}

$\mathrm{La}$ " civilisation paroissiale » repose sur des pratiques communes, une homogénéité des pratiques qu'il est difficile de surmonter. Les récits abondent d'innovations pastorales ou liturgiques contre lesquelles se sont dressées des oppositions malheureuses. Devant les nouveaux défis initiatiques et catéchétiques qui se posent à nous, peutêtre faudrait-il désigner des lieux laboratoires comme tels. En plus de faire connaître et analyser les success stories qui ne manquent pas dans nos communautés québécoises.

Les dérapages que nous avons connus dans le cadre de réformes hâtives et d'initiatives éclatées, ne font que rappeler cette loi profonde de l'expérience religieuse, à savoir le besoin d'un minimum de consensus pour que les gens s'y retrouvent :

"Pour que le rite fonctionne bien, il faut un accord à peu près consensuel autour de lui. Dans les tentatives pour inventer des rituels, de nombreux ratés se produisent quand les gens ne se comprennent pas: souvent une partie du groupe voit dans la tentative de ritualisation et d'institution de gestes symboliques une marque d'emprise ou un fauxsemblant illusoire, et le geste symbolique manque son objectif. Sans un certain accord, le rite ne remplit pas son office ... la question de la croyance est alors importante dans le groupe, et bien souvent il va falloir donner un nouveau sens à des formes anciennes pour que chacun s'y retrouve $^{26} »$.

Pourquoi ne pas créer de petits "instituts de recherche " ecclésiaux, en lien avec les diocèses et les facultés et départements de théologie ? Les divers départements universitaires ont toujours des lieux de recherche désignés et institués. Or, le lien analytique et créateur aux milieux ecclésiaux est surtout laissé à la libre appartenance ou initiative des universitaires ou autres chercheurs, prêtres, religieux ou laïcs. Peut-on constituer plus systématiquement ces lieux de collaboration et d'expérimentation ciblés ?

26. Jean LEMAIRE, «Des rites de passage qui ne disent pas toujours leur nom ", dans Dialogue. Recherches cliniques et sociologiques sur le couple et la famille, no $127,1^{\text {er }}$ trimestre 1995, p. 6. 


\subsection{Un enjeu d'incorporation: la pertinence du groupe dans lequel}

nous sommes initiés et incorporés

L'un des aspects de l'initiation est l'entrée dans un groupe social ou religieux. Or, de manière diffuse, les contentieux du monde moderne avec le catholicisme se répercutent dans le rapport des Québécois et des Québécoises avec l'Église catholique : on y entre, mais sous condition. On y entre à divers titres, on refuse d'y entrer à d'autres titres. Et souvent, on se refuse à une pleine incorporation dans le corps ecclésial. Certes, ce quant-à-soi réfère à des conditions sociologiques d'appartenance beaucoup plus individualisées, à une crise de l'engagement, et le reste et le reste. Mais il réfère aussi à de profonds et sérieux contentieux des citoyens modernes avec l'institution catholique. Il s'agit d'un constat assez banal et habituel dans notre milieu. Mais il m'amène à faire la proposition suivante :

On ne saurait négliger l'importance des actions et paroles des représentants ecclésiaux locaux sur la place publique, comme médiation différenciée du Saint Siège romain. Durant les trente dernières années, l'épiscopat canadien et québécois a souvent adapté ou nuancé les positions romaines (qu'on pense aux débats délicats et houleux qui ont suivi la parution de l'encyclique Humanae Vitae). C'est le rôle fondamental d'une Église locale d'affirmer sa couleur propre. Et non seulement l'épiscopat canadien s'est-il en général présenté comme réformateur, mais il a reconnu une pluralité des voix en son sein. Or, le resserrement centralisateur du catholicisme durant les dernières années m'apparaît compromettre gravement l'écart très sain entre Saint Siège romain et Église locale en débat. Si cet écart continue de s'atténuer, on ne peut espérer une incorporation confiante et engagée d'un nombre significatif de membres des jeunes générations dans les années qui viennent. C'est en effet une caractéristique des jeunes générations de rechercher, à travers la fameuse quête d'authenticité, une adéquation entre positions personnelles et appartenance institutionnelle. Fidèle à sa tradition des dernières décennies, l'Église canadienne me semble détenir à ce titre un rôle réformateur fondamental dans le catholicisme, qu'elle devrait continuer de jouer avec audace. Car à l'horizon de l'initiation se trouve la grande assemblée des chrétiens et des chrétiennes.

Pour conclure, dans le nouveau contexte, les mois et les années d'investissements qui viennent en éducation de la foi seront le fruit 
d'un travail collectif. Cela va de soi. Mais il y a quelque chose de particulier : il s'agit d'un travail d'abord intergénérationnel. Qu'est-ce à dire ? De nombreux seniors qui ont participé au formidable mouvement catéchétique des années 1960 et 1970 nous rappellent présentement, lorsque nous les croisons, les questions, les explorations, les audaces et les défriches qui marquèrent ces années effervescentes. Nous ne partons certes pas à zéro. Des générations plus jeunes de théologiens et d'intervenants ecclésiaux relisent maintenant à nouveaux frais ces approches très riches. Au-delà des crises nombreuses qui secouent l'Église catholique québécoise, j'estime que ce travail d'interprétation collectif peut être fécond et dynamisant pour nos milieux, car il fera à la fois œuvre de mémoire et d'innovation.

\begin{abstract}
RÉSUMÉ
Comment réfléchir aujourd'hui sur l'initiation en christianisme ? L'anthropologie renvoie à un sens de l'initiation plus précis que ne le fait le champ de l'initiation sacramentelle, très large. Et ce sens fondamental se rapporte notamment à la distinction entre rites d'initiation et rites de passage. Les premiers se fondent dans le processus d'incorporation proprement rituelle. Cela dit, il reste que l'approche de l'initiation dans nos sociétés modernes se présente plus généralement comme un chemin de sens évoquant de loin et par traces les antiques expériences initiatiques. L'enjeu est de saisir comment ces expériences continuent de travailler les quêtes religieuses et chrétiennes actuelles.
\end{abstract}

\title{
ABSTRACT
}

How can we reflect on initiation in Christianity? In anthropology, the meaning of the term initiation is far precise than in the much larger field of sacramental initiation. This fundamental meaning pertains to the distinction between initiation rituals and rites of passage, among others. The former are constructed in the process of the ritual incorporation. However, the approach to initiation in our modern societies is generally a way of making meaning, and it evokes only traces of the antique initiatory experiences. The challenge is to understand how these experiences continue to work on/to shape religious and Christian quests. 